\title{
Unusual Hyperechoic Appearance of Hodgkin's Lymphoma in the Liver
}

\section{Introduction \\ $\nabla$}

Liver lesions of Hodgkin's lymphoma and other lymphoma types usually appear as hypoechoic lesions in grayscale abdominal ultrasound. Here we describe the unusual case of hyperechoic liver nodules caused by Hodgkin's lymphoma.

\section{Case Description}

$\nabla$

A 40-year-old female presented at our hospital for work-up of a retroauricular ulcer that had persisted for weeks. She reported painless enlargement of cervical lymph nodes and significant weight loss during the last few months. Biopsy of the ulcer with subsequent pathological examination demonstrated a massive infiltration of T-cell dominating lymphocytes, granulocytes and plasma cells. Immunohistochemical examination of the lymphoproliferative infiltrates revealed the diagnosis of Hodgkin's disease.

Staging examinations including contrastenhanced computed tomography (CT) of the neck, chest and abdomen showed additional hypodense lesions in the liver and spleen ( $\boldsymbol{O}$ Fig. $\mathbf{1}$ ).

An ultrasound examination of these lesions in B-mode using an Acuson, Sequoia, 512 scanner (Siemens Medical Solution) revealed multiple hyperechoic masses in the liver up to $2 \mathrm{~cm}$ in size ( $\bullet$ Fig. 2 upper panels) and hypoechoic masses inside the spleen up to $8 \mathrm{~cm}$ in size ( $\bullet$ Fig. 2 lower panels). Contrastenhanced ultrasound of the liver lesions using Sonovue contrast medium (Bracco SpA, Milan Italy) revealed an isoechoic enhancement pattern compared to the surrounding liver tissue in the arterial phase. During the portal-venous and parenchymal phase, the lesions rapidly washed out the contrast medium and appeared hypoechoic in comparison to the surrounding liver tissue. Splenic lesions showed a pronounced homogenous hypoechoic enhancement pattern during all recorded phases compared to the surrounding splenic tissue. Due to the fact that lymphoma involvement of the liver usually does not present a hyperechoic pattern in B-mode and that the contrast-enhanced examination indicated malignant lesions, we performed a biopsy of one of the liver masses using a 18 -gauge needle with a throw of $33 \mathrm{~mm}$. Differential diagnosis of hyperechoic liver lesions include metastatic carcinoma and multifocal hepatocellular carcinoma. Both diagnoses were less likely because there were no known primary and no signs of liver cirrhosis present. Pathological examination revealed next to the normal liver tissue a lymphocytic infiltrate associated with formation of intracellular fat in the surrounding hepatocytes. The density of vessels indicated by immunohistochemistry using antibodies against CD31 and CD34 was increased in the infiltrated areas ( $\bullet$ Fig. 3). Further immunohistochemical analyses confirmed liver infiltrates of Hodgkin's lymphoma. Using the Ann Arbor Staging System with Cotswolds Modifications, the patient was staged as grade IV, BHS. Chemotherapy using the BEACOPP regimen (Cyclophsphamide, Adraimycine, Etoposide, Procarbacine, Prednisolone, Neulasta) was started. Follow-up examinations using PET-CT after two cycles of chemotherapy presented regression of all lesions including the cervical lymph nodes, liver and spleen masses.

\section{Discussion}

$\nabla$

Hodgkin's lymphoma accounts for approximately $10 \%$ of all lymphomas. The incidence in Europe is about 2.4 cases per 100000 persons (Sant $M$ et al. Blood. 2010 Nov; 116(19): 3724-3734.). Liver involvement of lymphomas in general can be found in up to $50 \%$ of cases (Castroagudin JF et al. J Ultrasound Med. 2007 Jun; 26(6): 791-796.). The vast majority of sonographic findings are lesions with a hypoechoic appearance. A small portion of cases present with a heterogeneous sonographic appearance of the focal lesions (Soyer $P$ et al. Abdom Imaging 1993;18(4): 339-343.). Here we present the first detailed report to our knowledge of a Hodgkin's lymphoma presenting as hyperechoic liver lesions. This case was part of a cumulative analysis of the contrast-enhanced ultrasound pattern of hepatic lymphoma (Trenker C et al. Ultraschall Med. 2014 Apr; 35(2): 142-148.).

Increased echogenicity of liver lesions has been attributed to fat deposition (Caturelli E et al. Gastroenterology 1991; 100(6): 1678-1682.). One form, the fatty liver disease also known as nonalcoholic fatty liver disease, can present as patchy or focal hyperechoic areas inside the liver (Gorg C et al. Onkologie 2005; 28(12): 659-664.). Other examples of hyperechoic liver lesions include liver adenomas. Liver adenomas with a hyperechoic appearance can also reveal intratumoral fat deposition in histological analysis which is the main factor contributing to the echogenicity (Hung $\mathrm{CH}$ et al. Abdom Imaging. 2001 Sep-Oct; 26(5): 500-506.). Additional lesions presenting with a hyperechoic pattern are hemangiomas. Increased vessel density contributes in these cases mainly to the high echogenic-

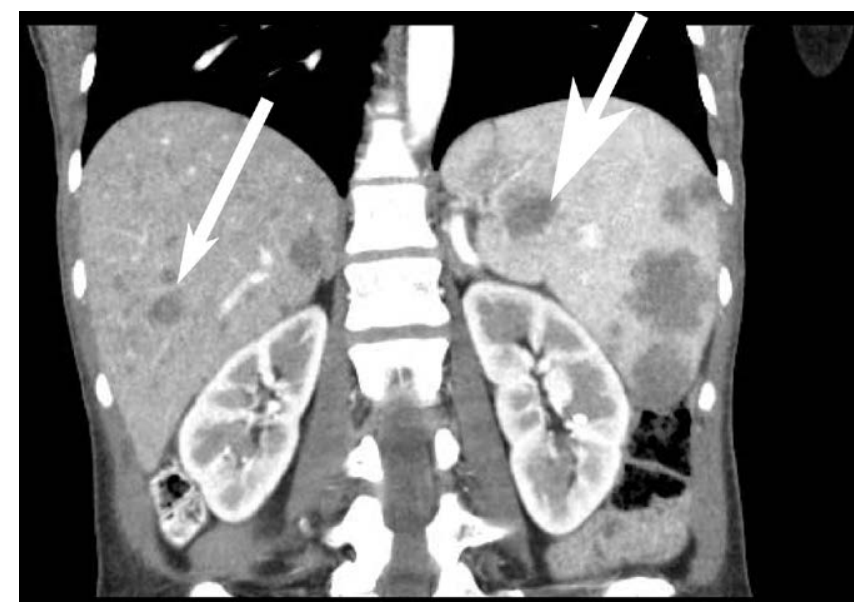

Fig. 1 Contrastenhanced CT scan in longitudinal section reveals multiple hypodense lesions of the liver (small arrowhead) and spleen (large arrowhead). 


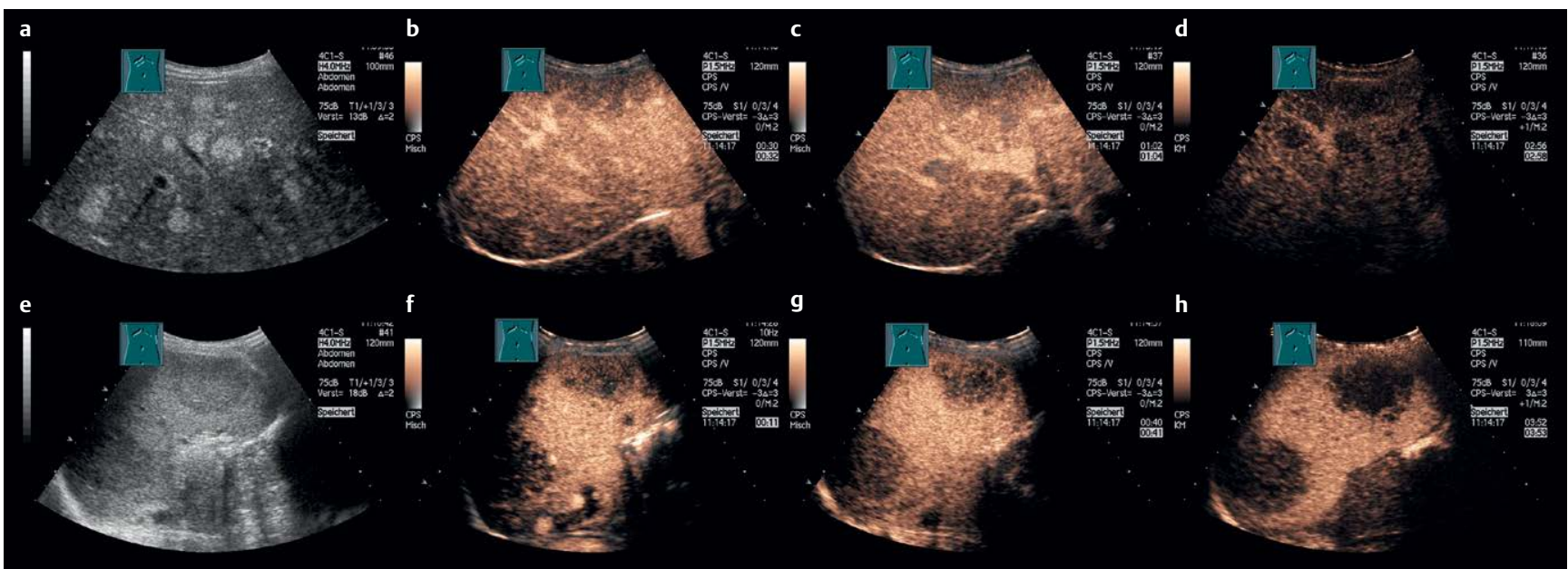

Fig. 2 Contrast-enhanced ultrasound of liver (upper panels) and spleen (lower panels) lesions. Baseline ultrasound of the liver a and spleen e followed by the contrast-enhanced arterial phase $\mathbf{b}, \mathbf{f}$, portal venous phase $\mathbf{c}$ or parenchymatous phase $\mathbf{g}$ and late parenchymatous phase $\mathbf{d}$, $\mathbf{h}$ demonstrates the characteristics of the lesions.

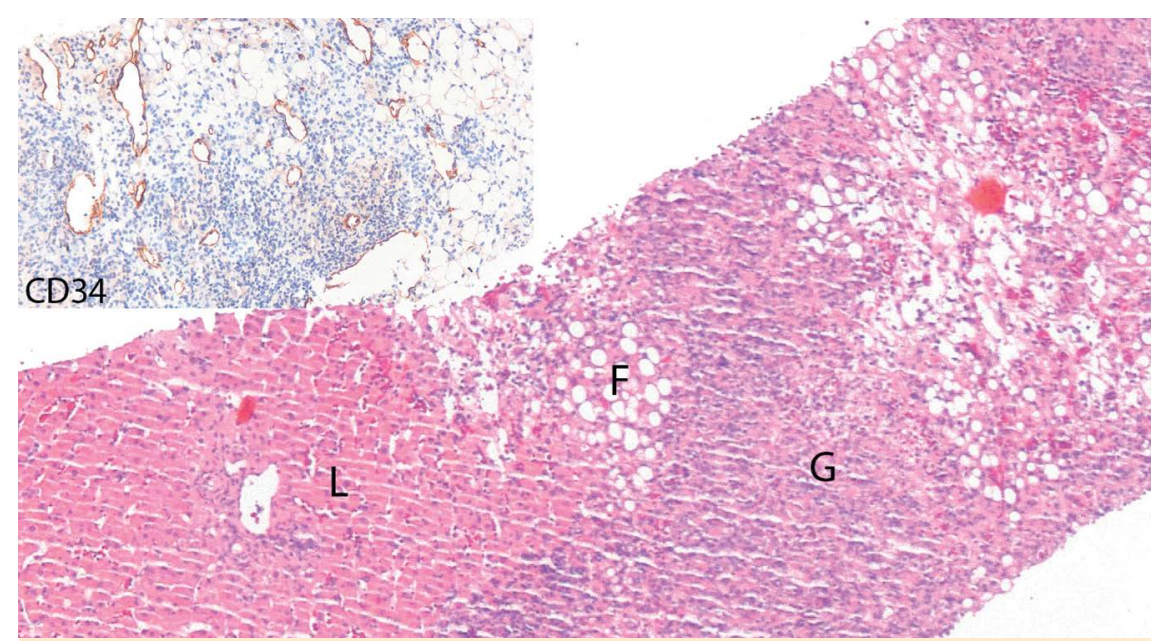

Fig. 3 Liver biopsy of 40-year-old female patient with dense lymphoid infiltrate between macrovesicular steatosis (F). The lymphoid infiltrate with partly granulomatous pattern (G) and corresponding to an infiltration of the Hodgkin's lymphoma. To the left, inconspicuous liver tissue (L). The small inset depicts immunohistochemistry for the endothelial marker CD34 with small to medium-sized capillaries in the lymphoma infiltrate.

ity of the lesions. In the case of the hyperechoic lesions presented by our patient, histopathological work-up revealed fat deposition in hepatocytes surrounded by immune cell infiltrates. The adjacent normal liver parenchyma presented no fatty deposition. This supports the hypothesis that fat deposition is responsible for the echogenicity. Additionally, small to medium-sized vessels may have contributed to the hyperechogenicity. A thorough review of the literature revealed a few case reports of lymphomas that have been associated with liver masses with a heterogeneous appearance on ultrasound (Castroagudin JF et al. J Ultrasound Med.
2007 Jun; 26(6): 791-796., Wernecke K et al. Br J Radiol. 1987 Jul; 60(715): 655660.). Unfortunately no correlation between the sonographic appearance and the presented histopathological picture was made. The most similar description of a hyperechoic non-Hodgkin's lymphoma liver mass was written by Kim and colleagues (Kim $\mathrm{H}$ et al. J Clin Ultrasound 2008; 36(7): 437-439.). The authors described an echogenic mass that infiltrated the liver. Fat deposition was attributed to the echogenicity of the lesion. The pathological specimen displayed adipose tissue diffusely merged with hepatocytes and immune cells. In contrast, our patient displayed the fat deposition inside the hepatocytes surrounded by lymphoma infiltrates. Degenerative fat accumulation inside the hepatocytes triggered by the lymphoma may be the cause for this phenomenon. Further investigations are necessary to correlate the sonographic appearance of liver lesions to the histopathological characteristics of the tissue.

A. Hann, C. Trenker, C. C. Westhoff, C. Goerg, Marburg 